\title{
EDITORIAL
}

\section{Vascular surgery in Canada}

York N. Hsiang*

\section{Demographics}

Following the dissolution of the Union of Soviet Socialist Republics in 1991, Canada became the world's largest country land mass. Comparatively, its population is very sparse at approximately 33 million, and distributed mainly along the border with the United States.

Canada consists of ten provinces and two territories, governed by a constitutional monarchy. Although English and French are the two national languages, English is predominant in every province, except in Quebec, where French is spoken almost exclusively.

\section{Canadian Health care system}

The Canadian Health care system was originally introduced in 1962 by Tommy Douglas, a politician of the New Democratic Party in the province of Saskatchewan. Based on the tenets of being universal, accessible, comprehensive, portable, and publicly administered, it has maintained its original form for the past four decades. It covers all medically necessary procedures, including expensive ones, such as transplantation and dialysis. However, dental care, optometry, and most outpatient prescription drugs are not covered. Patients who require inpatient care have all their medications, investigations, and procedures covered. Despite this, it has been estimated that $30 \%$ of Health care (primarily for dental care, optometry and prescription drugs) is privately funded through private insurance companies or directly paid by patients. Private surgical clinics exist for basically short stay cosmetic or worker's compensation procedures.

The cost to run such a Health care system is covered primarily by taxation either directly or indirectly. Employees of corporations may make payments through payroll deductions. Other privately employed adults make payments directly to the provincial government. Only those at or below the poverty level of income pay no Health care fees, but still receive comprehensive coverage.

Canada currently spends approximately $10 \%$ of its gross domestic product (GDP) on its Health system. In contrast, the United States spends 16\% of its GDP and Brazil, 7.9\%. Despite this, the Canadian Health care system serves its population well based on crude measurements such as longevity (average life expectancy is 81.3 years) and infant mortality (4.5/1,000 live births).

Canadians are generally satisfied with the Health care they receive. It has been estimated through public opinion polls that $70 \%$ of the Canadian people is satisfied with the Health care system. Despite this, the primary criticisms of the system are long waiting lists for specialist physicians, expensive diagnostic testing - computerized tomography (CT) or magnetic resonance imaging (MRI) scans - and elective surgery.

\section{History of vascular surgery in Canada}

Much like other countries, the subspecialty of Vascular Surgery grew out of General Surgery. Those general surgeons who were interested in this challenging new area established the Canadian Society for Vascular Surgery (CSVS), with the stated objectives of providing a forum for surgeons to discuss vascular cases, improve standards of care, establish educational standards, and promote research progra$\mathrm{ms}$ in vascular disease in Canada. The founding meeting was in 1978, and the first examination, conducted through The Royal College of Physicians and Surgeons of Canada, was in 1983. Current candidates have to initially receive certification in General Surgery and satisfactorily complete a Vascular Surgery Fellowship to qualify for this examination (called Certificate of Special Competence in Vascular Surgery). Once qualified in Vascular Surgery, most surgeons perform only this subspecialty, although almost $25 \%$ of vascular surgeons still do General Surgery. 


\section{Training of vascular surgeons}

Vascular Surgery Fellowship currently requires two extra clinical years of training following General or Cardiac Surgery. During this time, trainees are exposed to both open and endovascular training. Currently, there are ten Vascular Surgery training programs in Canada, and each centre trains up to two fellows annually. Starting in 2012, many Vascular Surgery training programs may also offer a direct path. Thus trainees, in addition to the traditional track, may enter Vascular Surgery directly from Medical School rather than complete a General Surgery residency first. The expected time to complete a Vascular Surgery residency, directly from medical school, is five years.

\section{Vascular Surgical practice}

Nowadays, there are approximately 150 members of the CSVS. At the most recent CSVS annual meeting, 70\% of the attendees indicated that they currently perform endovascular procedures. This proportion will probably increase in the future due to attrition of older members who do not perform endovascular procedures. The majority of endovascular procedures are performed in the operating room with mobile $\mathrm{C}$-arm units, although more hospitals are planning on constructing hybrid operating rooms with fixed unit image intensifiers.

As a result of the publicly funded Health care system, all physicians bill the government Health insurance plan directly. This simplifies administration and, as a result, minimizes competitive practices such as advertising. All patients request to see a specialist physician. However, they must be referred by another physician.

Standards of care including registration are overseen by provincial Colleges of Medicine. Of all the different Health care systems that the author has witnessed, one of the main benefits to patients who require surgical care is that, in the Canadian Health care system, consultant care is the standard - even for the simplest incisional and drainage procedure. Rarely patients are operated on by unsupervised residents or fellows.

Medical indemnity in Canada is handled by a single not-for-profit insurance company, the Canadian Medical Protective Association. All Canadian physicians are members of this well run association. Fees vary according to the province and area of Medicine practised. Compared to the United States, these fees are very low. Furthermore, as it is a not-for-profit association, there is usually a partial rebate of annual fee monies after expenses. For instance, in the province of British Columbia, Vascular Surgery carries a risk level of 5 (the maximum is risk level 6 for obstetricians, neurosurgeons, and cardiac surgeons). After rebates for the annual premium, the expected annual fee would be no more than CAD $\$ 3000$.

On a day-to-day level, physicians practice the art and science of Medicine and Surgery with the best of their abilities. Care maps and rigid admission and discharge criteria are not common. The majority of physicians are paid for service, although alternative payment models, such as service contracts or other forms of salary, are common.

In summary, the Canadian Health care system has well served Canada and its physicians. There are ongoing issues related to funding that leads to wait lists and lack of hospital resources (such as operating room time) but, overall, the majority of citizens and physicians are satisfied with their Health care system.

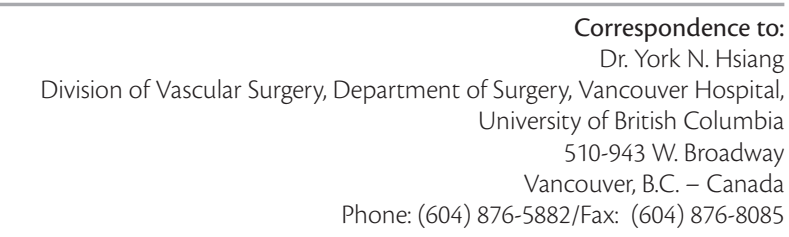

